\title{
Eye Movement Desensitization and Reprocessing to Facilitate Posttraumatic Growth: A Prospective Clinical Pilot Study on Ferry Disaster Survivors
}

\author{
Sang Won Jeon ${ }^{1}$, Changsu Han ${ }^{2}$, Joonho Choi ${ }^{3}$, Young-Hoon Ko ${ }^{2,4}$, Ho-Kyoung Yoon ${ }^{2}$, Yong-Ku Kim ${ }^{2}$ \\ ${ }^{1}$ Department of Psychiatry, Kangbuk Samsung Hospital, Sungkyunkwan University School of Medicine, Seoul, ${ }^{2}$ Department of Psychiatry, Korea \\ University Ansan Hospital, Korea University College of Medicine, Ansan, ${ }^{3}$ Department of Psychiatry, Hanyang University Guri Hospital, Hanyang \\ University College of Medicine, Guri, ${ }^{4}$ Ansan Mental Health Trauma Center, Ansan, Korea
}

\begin{abstract}
Objective: The purpose of this study was to investigate the therapeutic effects of eye movement desensitization and reprocessing (EMDR) on post-traumatic growth (PTG).

Methods: This study was conducted using a sample of ten survivors of a large-scale maritime disaster that occurred in the Yellow Sea, South Korea, in April 2014. A total of eight EMDR sessions were administered by a psychiatrist at two-week intervals over a period of five months, starting two or three months after the accident. Post-Traumatic Growth Inventory (PTGI), Stress-Related Growth Scale (SRGS), Clinician-Administered PTSD Scale (CAPS), and Connor-Davidson Resilience Scale (CD-RISC) were measured before treatment, after sessions 4 and 8 , and at three months after treatment completion.

Results: After three months from treatment completion, significant increases were observed in PTG (PTGl: $Z(8)=-2.380, p=0.017$; SRGS: $Z(8)=-2.380, p=0.017)$ and resilience (CD-RISC: $Z(8)=-2.386, p=0.017)$. A decrease in post-traumatic stress disorder (PTSD) level was also significant (CAPS: $Z(8)=-2.176, p=0.030)$. The reduction of CAPS scores was correlated with increases of PTGl (rho $=0.78, p=0.023$ ) and SRGS (rho=0.79, $p=0.020$ ) scores. The changes in CAPS, PTGl, and SRGS scores between time point of end 8-session and three months follow-up was not significant (all $p>0.05$ ). Subjects with higher pre-treatment CD-RISC scores showed more significant improvements in PTGI (rho $=0.88, p=0.004$ ) and SRGS (rho $=0.83, p=0.010$ ) scores after treatment than did those with lower pre-treatment CD-RISC scores.

Conclusion: EMDR therapy using standard protocol for trauma processing helped facilitating PTG in disaster survivors. To generalize these findings, further controlled studies comparing with other treatment modalities for PTSD are needed.
\end{abstract}

KEY WORDS: Eye movement desensitization reprocessing; Post-traumatic growth; Post-traumatic stress disorders; Resilience; Trauma.

\section{INTRODUCTION}

Most approaches to human post-traumatic responses are focused on the negative sequelae of trauma, such as post-traumatic stress disorder (PTSD). ${ }^{1)}$ However, this approach can result in misinterpretation of post-traumatic responses and neglect of positive changes. Individual positive post-traumatic changes have been studied since the mid-1990s. ${ }^{2)}$ Post-traumatic growth (PTG) is defined as 'positive change experienced as a result of the struggle with trauma which emphasizes the transformative poten-

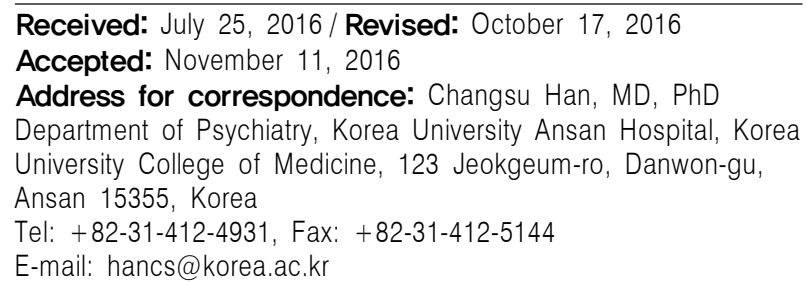

tial of one's experiences with highly stressful events and circumstances'. ${ }^{2)}$ Growth indicates not only recovery to the level before the trauma, but positive and new changes that exceed the previous level. ${ }^{3)}$ Accordingly, the scope of studies on psychological trauma has expanded from distress reduction to PTG facilitation, ${ }^{4)}$ but the application of PTG to clinical cases has been limited.

Most previous studies were not designed to facilitate PTG as primary goal, and it is rarely applied individually. In a randomized control study for women with nonmetastatic breast cancer, the experiment group $(n=58)$ who had completed an 8-session group intervention showed better primary outcome of PTG than the control group ( $\mathrm{n}=147)$ at 6 and 12 months following the baseline assessment. $^{5)}$ In a recent randomized controlled trial of cognitive-behavioral conjoint therapy for PTSD versus waitlist (40 couples), subjects who received treatment im-

(a) This is an Open-Access article distributed under the terms of the Creative Commons Attribution Non-Commercial License (http://creativecommons.org/licenses/by-nc/4.0) which permits unrestricted non-commercial use, distribution, and reproduction in any medium, provided the original work is properly cited. 
mediately demonstrated a significant increase in PTG. ${ }^{6)}$

Eye movement desensitization and reprocessing (EMDR) is a short-term, structured, eight-step therapy that is usually administered for six to 12 sessions, requiring approximately 90 minutes per session. ${ }^{7)}$ Images, cognition, emotions, and body sensations associated with traumatic memories are evaluated, and subjects are briefly exposed to traumatic memories while moving their eyes from side to side with free association.

Therapeutic effects of EMDR on PTSD have been reported because EMDR was initially developed to treat traumatic memories. ${ }^{8)}$ Traumatic memories represent incompletely processed information that triggers psychopathological effects. ${ }^{7)}$ Memories of past experiences, which can induce current negative cognition, emotions, and body sensations, are included in traumatic memories, although the trauma may not be severe enough to induce PTSD. Recently, EMDR has been used to treat subjects with traumatic memories, and has also been shown to be effective for subjects without PTSD. ${ }^{9,10)}$

The effects of EMDR therapy partly coincide with those of the PTG concept. EMDR helps transfer traumatic memories to semantic memories and helps subjects understand the meaning of their traumatic experiences and make sense out of them. ${ }^{7)}$ During EMDR therapy, 'ripple effects' often occur, meaning that the subject becomes free from traumatic memories, voluntarily performs new adaptive behaviors, and improves beyond the pre-traumatic state by showing improvements in symptoms. ${ }^{11)}$ During EMDR therapy, subjects experience not only simple recovery of traumatic events, but also frequently experience self-transcendence. This indicates that positive post-traumatic changes and growth can occur during EMDR sessions. Unlike cognitive behavior therapy (CBT), voluntary psychological association is emphasized in EMDR, which implies that the therapeutic results of EMDR do not follow the CBT model of habituation. ${ }^{12)}$ Patients not only re-interpret the meaning of their traumatic memories, but may experience new insights and adaptive thoughts spontaneously emerged by free association during EMDR therapy.

EMDR is an integrated psychotherapy that includes various psychotherapeutic theories. Clinicians recognize that a psychodynamic approach is possible during EMDR sessions, and this is confirmed through free association, symbolic expression, abreaction, and dealing with family issues. ${ }^{13)}$ In addition, a cognitive behavior therapeutic approach is applied during EMDR sessions and confirmed through learning processes, generalization, conditioned re- sponses, and positive changes from negative cognition. ${ }^{12,13)}$ EMDR is also a comparatively safe, non-invasive treatment method. These valuable characteristics of EMDR were considered appropriate for facilitating PTG.

Current clinical approaches for psychological trauma include not only PTSD treatment, but also PTG facilitation. $^{3,4)}$ PTG is important for post-traumatic subjects to help with long-term adaptation and promotes psychological and physical health. It is important to identify clinical methods suitable for facilitating PTG. EMDR has been used for PTSD treatment and other clinical applications. EMDR principles are well-adjusted for trauma treatments and are closely related to PTG concepts.

Most previous studies reported using EMDR to treat PTSD and reduce symptoms associated with trauma. There has been no research on EMDR and PTG, and it is unknown whether EMDR can facilitate and/or improve PTG. We investigated the clinical application of PTG in a group of subjects who had experienced the same trauma by analyzing changes after EMDR treatment.

\section{METHODS}

\section{Subjects}

In this prospective clinical pilot study, ten survivors of a large-scale maritime disaster that occurred in the Yellow Sea, South Korea, in April 2014 were targeted. All subjects were rescued from the same wreckage on the same day and voluntarily visited our psychiatric clinic after the accident. They did not specify EMDR as their preferred treatment method, but accepted EMDR after it was recommended.

The exclusion criteria were as follows; concomitant psychotropic medication, other forms of psychotherapy, additional treatment received from other institutions (for example, school counselling service), death of lineal family members or relatives in this maritime disaster (potential subject with complicated brief), history of organic mental disorders, alcohol or substance abuse, cognitive or memory impairments, and acute psychotic state.

This study was approved by the Institutional Review Board of Korea University Ansan Hospital (AS14132). All subjects provided written informed consent.

\section{Therapist}

A single psychiatrist (the first author) performed EMDR on the ten subjects, and the major supervisor (the corresponding author) supervised the study. The administering clinician completed a training course (EMDR part I 
and II) officially recognized by the EMDR International Association.

\section{Treatment}

The first EMDR sessions were administered two or three months after the accident, and all subjects underwent 8 sessions over a period of five months. Sessions were administered at two-week intervals, and each session was 90 minutes long. A 60-minute information-gathering session was held before the first session, during which the rationale for the procedure was explained to the subjects.

The treatment was not designed specifically for PTG facilitation, but for regular EMDR processes as part of Shapiro's procedures, ${ }^{7)}$ which include: 1) history taking and establishment of a treatment plan, 2) pre-treatment preparation, 3) assessment, 4) desensitization, 5) installation, 6) body scan, 7) closure, and 8) re-evaluation. A typical EMDR session begins with assessment phase where subject's specific traumatic memory is evaluated. Elements of traumatic memory are sought in order of image, negative belief, substitutable positive cognition, emotion, and body sensation. And while the patient is holding these elements of memory in mind, the therapist asks the patient to perform horizontal eye movement and follow up the patient's spontaneous associations at regular intervals, usually around 23 sets of eye movements. During these processes, unpleasant emotions and body sensations were also processed.

\section{Measurement Instruments}

Post-Traumatic Growth Inventory (PTGI), Stress-Related Growth Scale (SRGS), Clinician-Administered PTSD Scale (CAPS), and Connor-Davidson Resilience Scale (CD-RISC) were measured before treatment, after sessions 4 and 8 , and at three months after treatment completion. PTGI and SRGS were measured to evaluate PTG improvement. CAPS was measured to evaluate PTSD level, and CD-RISC was measured to check resilience. CAPS was the only metric assessed by the clinician, while the other measures were self-reported.

\section{Post-Traumatic Growth Inventory (PTGI)}

$\mathrm{PTGI}^{2)}$ is an instrument for assessing positive outcomes reported by individuals who experience traumatic events. It is useful for determining how successful individuals will be at coping with life and for reconstructing or strengthening their perceptions of themselves, others, and the meaning of events after a trauma. This 21-item scale includes five factors: new possibilities, relating to others, personal strength, spiritual change, and appreciation of life. Each item is assigned 0-5 points. A high score indicates a high PTG level.

\section{Stress-Related Growth Scale (SRGS)}

SRGS $^{14)}$ quantifies positive outcomes of stressful events. SRGS items represent positive changes in personal resources, social resources, and coping skills. The single factor of growth includes 50 items. Each item is evaluated on a scale ranging from 0 to 2 . A higher score indicates improvement in growth associated with trauma.

\section{Clinician-Administered PTSD Scale (CAPS)}

CAPS $^{15)}$ is widely considered the gold standard in PTSD assessment. It is a structured interview that yields a categorical diagnosis, as well as a measure of the severity of PTSD symptoms as defined by the Diagnostic and Statistical Manual of Mental Disorders 4th edition (DSM-IV). It was designed to be administered by clinicians and clinical researchers with a working knowledge of PTSD. The full interview takes 45 to 60 minutes to administer. The Korean version of CAPS has proven to have excellent reliability and relevance (Cronbach's $\alpha=0.95)$. ${ }^{16)}$

\section{Connor-Davidson Resilience Scale (CD-RISC)}

$\mathrm{CD} \mathrm{RISC}^{17}$ is a brief self-rating questionnaire that measures resilience. It was developed as a measure of stress coping ability for clinical practice. It includes five factors (personal competence, trust/tolerance/strengthening effects of stress, acceptance of change and secure relationships, control, and spiritual influences) and 25 items. Each item is assigned 0 to 4 points. A higher score indicates higher resilience. The Korean version of CDRISC has been validated (Cronbach's $\alpha=0.93$ ). ${ }^{18)}$

\section{Data Analysis}

The Wilcoxon signed rank test was conducted to determine the therapeutic effects of EMDR, and the effect size (ES) was calculated to compare the effects in each field after EMDR therapy. Spearman's rank order correlation test was used to assess the correlation between reduction in PTSD level and increase in PTG. Correlation test was also used to assess the relationship between pre-treatment resilience level and improvement in PTG after treatment. All analyses were conducted using IBM SPSS Statistics ver. 20.0 for Windows (IBM Co., Armonk, NY, USA). All statistical tests were two-tailed. 
Table 1. Subject demographics, information about inpatient treatment, and DSM-5 diagnoses at the beginning of EMDR

\begin{tabular}{rcccc}
\hline Patient & Sex & Age $(\mathrm{yr})$ & Admission (hospitalized day) & Diagnosis at the beginning of EMDR \\
\hline Patient 1 & M & 42 & No & Partial PTSD \\
Patient 2 & M & 18 & 14 & Partial PTSD \\
Patient 3 & F & 18 & 14 & Partial PTSD \\
Patient 4 & F & 18 & 14 & Partial PTSD \\
Patient 5 & M & 58 & 21 & Partial PTSD \\
Patient 6 & F & 18 & 14 & PTSD \\
Patient 7 & M & 18 & 14 & Partial PTSD \\
Patient 8 & F & 38 & No & PTSD, MDD \\
Mean (SD) & & $28.5(15.6)$ & $15.2(2.9)$ & \\
\hline
\end{tabular}

DSM-5, the Diagnostic and Statistical Manual of Mental Disorders 5th edition; EMDR, eye movement desensitization and reprocessing; M, male; F, female; PTSD, post-traumatic stress disorder; MDD, major depressive disorder; SD, standard deviation.

Table 2. Changes in mean scores of eight patients for each scale

\begin{tabular}{|c|c|c|c|c|}
\hline Variable & CD-RISC & CAPS & PTGI & SRGS \\
\hline Pre-session & $50.0(15.8)$ & $49.1(11.1)$ & $46.8(8.2)$ & $44.5(6.4)$ \\
\hline After session 4 & - & $41.0(18.0)$ & $54.9(11.8)$ & $54.9(10.7)$ \\
\hline After session 8 & $57.6(18.3)$ & $37.6(17.7)$ & $62.1(13.4)$ & $62.3(14.1)$ \\
\hline Three months after treatment completion & $55.1(18.7)$ & $37.9(20.6)$ & $62.1(16.8)$ & $61.4(16.4)$ \\
\hline Statistics from pre- to post-treatment & $\begin{array}{c}Z(8)=-2.386^{* *} \\
p=0.017\end{array}$ & $\begin{array}{c}Z(8)=-2.176^{*} \\
p=0.030\end{array}$ & $\begin{array}{c}Z(8)=-2.380^{* *} \\
p=0.017\end{array}$ & $\begin{array}{c}Z(8)=-2.380^{* *} \\
p=0.017\end{array}$ \\
\hline Increase and decrease rates (\%) & +10.2 & -22.8 & +32.7 & +38.0 \\
\hline Effect size & 0.30 & 0.71 & 1.22 & 1.48 \\
\hline
\end{tabular}

Values are presented as mean (standard deviation).

CD-RISC, Connor-Davidson Resilience Scale; CAPS, Clinician-Administered PTSD Scale; PTGI, Post-Traumatic Growth Inventory; SRGS, Stress-Related Growth Scale.

Statistic: Wilcoxon signed rank test from pre- to post-treatment; ${ }^{*} p<0.05,{ }^{* *} p<0.025$.

\section{RESULTS}

\section{Study Sample Analysis}

Subjects initially consisted of three adults and seven high school students. All seven students were attending the same school and were the same age (18 years) and grade. One student dropped out at EMDR session 3 because he moved to a new residence. The other student changed her mind about receiving psychotherapy every week at EMDR session 4, and her therapist was changed; she preferred meeting the clinician for briefer periods of time on a more frequent basis. Per protocol analysis was performed because the data for post-treatment assessment in these two students were not collected. Finally, the remaining eight subjects who completed treatment through EMDR session 8 were analyzed in this study.

Table 1 shows the demographics of subjects, information about inpatient treatment, and the Diagnostic and Statistical Manual of Mental Disorders 5th edition (DSM-5) diagnoses at the beginning of EMDR. Four subjects were male and four were female, and their mean age was 28.5 years (standard deviation [SD], 15.6; range, 18-58). Six received inpatient treatment for a mean of 15.2

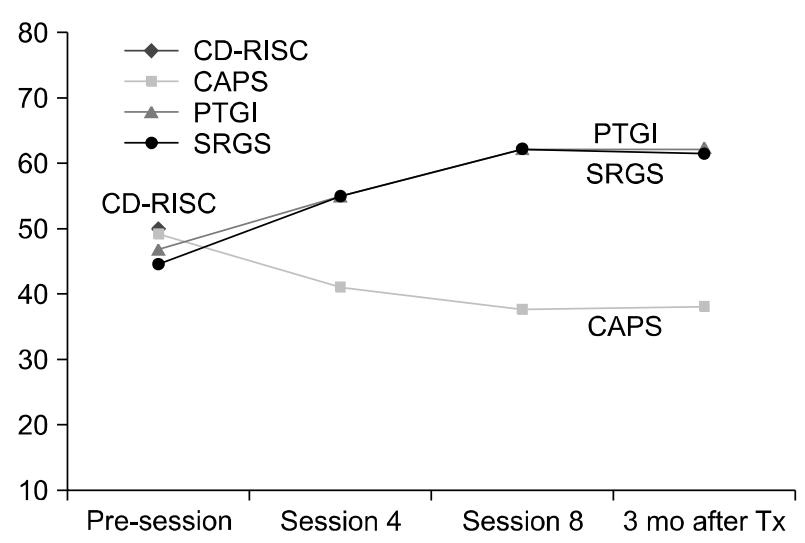

Fig. 1. Changes in mean scores for each scale from pre-treatment to three months after treatment completion $(n=8)$.

CD-RISC, Connor-Davidson Resilience Scale; CAPS, ClinicianAdministered PTSD Scale; PTGI, Post-Traumatic Growth Inventory; SRGS, Stress-Related Growth Scale; Tx, treatment.

days immediately after the accident. Patients were diagnosed on the basis of an unstructured clinical interview for DSM- 5 by a psychiatrist who performed EMDR. All subjects had initial diagnoses of acute stress disorder immediately after the accident. At two or three months after the 
accident when EMDR therapy was started, two subjects (patients 6 and 8) were diagnosed with PTSD. Patient 8 had a comorbid major depressive disorder (MDD). The remaining patients were diagnosed with partial PTSD (Table 1). After three months from treatment completion, one of the subjects (patient 8) retained the diagnosis of PTSD and MDD.

\section{Treatment Outcomes of All Subjects}

Table 2 and Figure 1 show the changes in mean scores for each scale at the pre-EMDR session, after sessions 4 and 8, and three months after EMDR therapy completion.
Table 2 also shows the results of therapeutic effect examinations, increases and decreases in rates $(\%)$, and ESs. The changes for each subject are shown in Figure 2.

Wilcoxon signed rank tests revealed significant improvements from pre-treatment to three months after treatment completion for all measures. After EMDR therapy, increases were observed in the PTG-associated PTGI $(Z(8)=-2.380, p=0.017)$ and SRGS $(Z(8)=-2.380, p=$ $0.017)$ scores and in the resilience-associated CD-RISC score $(Z(8)=-2.386, p=0.017)$. A decrease in the PTSD-associated CAPS score was also observed $(Z(8)=$ $-2.176, p=0.030)$. The reduction of CAPS scores was
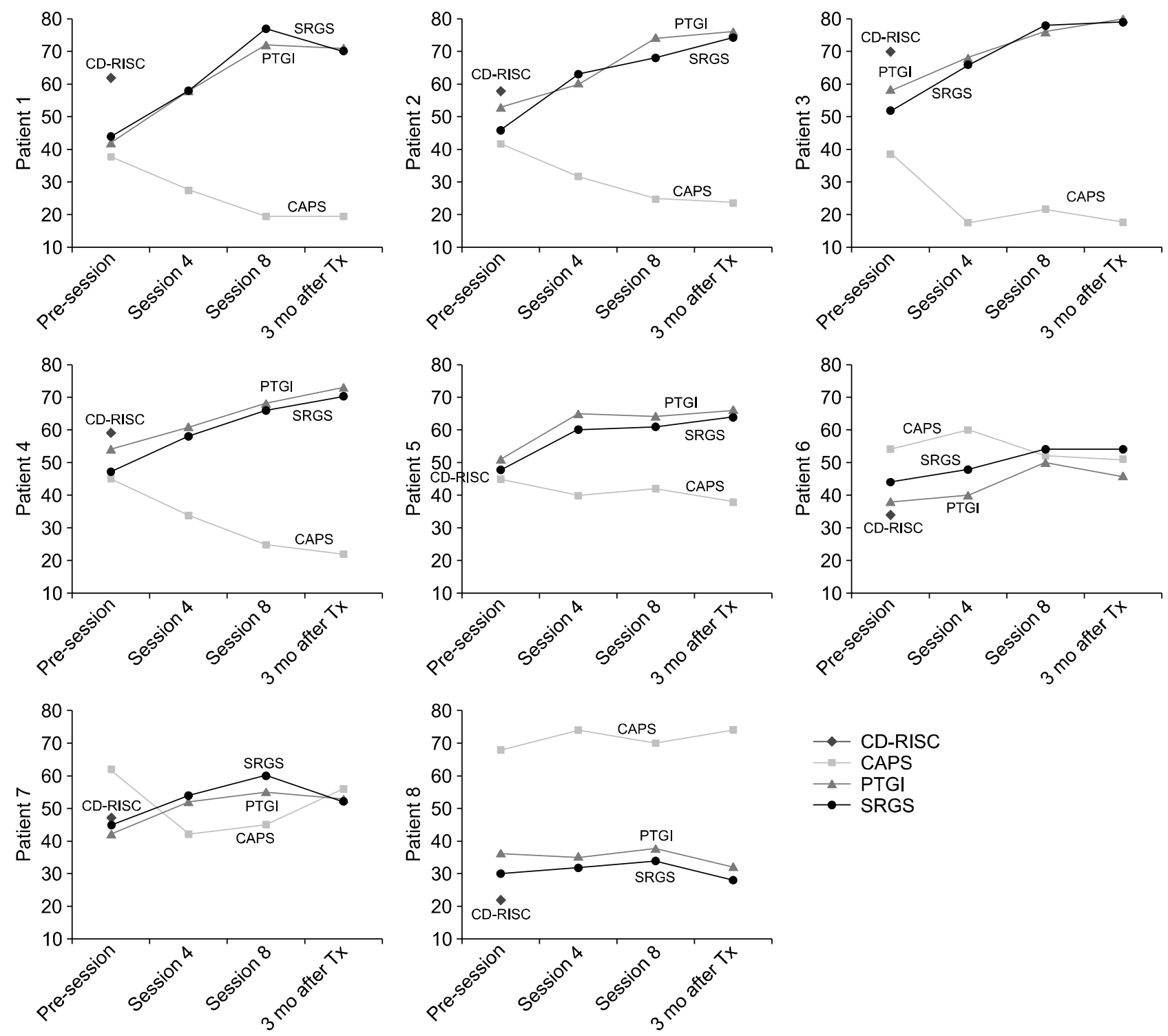

Fig. 2. Changes in scores of each patient for each scale from pre-treatment to three months after treatment completion. CD-RISC, Connor-Davidson Resilience Scale; CAPS, Clinician-Administered PTSD Scale; PTGI, Post-Traumatic Growth Inventory; SRGS, StressRelated Growth Scale; Tx, treatment. 
correlated with increases of PTGI (Spearman's rho $=0.78$, $p=0.023)$ and SRGS (rho $=0.79, p=0.020)$ scores. The PTGI (ES=1.22) and SRGS (ES=1.48) ES were larger than those of CAPS (ES=0.71) and CD-RISC ( $E S=0.30)$.

The changes in CAPS, PTGI, and SRGS scores between time point of end 8-session and three months follow-up was not significant (CAPS: $Z(8)=-0.341, p=0.733$; PTGI: $Z(8)=-0.070, p=0.944$; SRGS: $Z(8)=-0.593, p=0.553)$.

\section{Case Series Analysis}

During the EMDR sessions, PTGI and SRGS gradually increased in all subjects except patient 8 . At three months after treatment completion, these seven subjects showed increases in PTGI, SRGS, and CD-RISC scores. The CAPS score showed an inconsistent pattern, decreasing significantly (therapeutic response was defined as CAPS reduction $\geq 30 \%{ }^{15}$ ) in Patients 1, 2, 3, and 4 (decrease rates: $47 \%, 43 \%, 54 \%, 51 \%$, respectively), barely decreasing in patients 5,6 , and 7 (decrease rates: $16 \%, 6 \%$, $10 \%$, respectively), and increasing in patient 8 (increase rate: $9 \%$ ).

At three months after treatment completion, patients 1, 2, 3, and 4 showed significant decreases in CAPS score and significant increases in PTGI. During EMDR therapy, PTSD was relieved and PTG was facilitated in these patients. Patients 5, 6, and 7 showed small decreases in CAPS score and comparatively large increases in PTGI score. After EMDR was completed, PTG was facilitated regardless of PTSD relief. For patient 8, the CAPS score did not decrease, but slightly increased (68 to 74 ), and the PTGI (36 to 32) and SRGS (30 to 28) scores slightly decrease.

In all subjects, higher pre-treatment CD-RISC scores were associated with more significant improvements in PTGI (Spearman's rho $=0.88, p=0.004$ ) and SRGS $(\mathrm{rho}=0.83, p=0.010)$ scores after treatment. Higher CD-RISC scores were also correlated with significant reduction in CAPS score (rho $=0.91, p=0.002$ ). When higher resilience was observed before EMDR therapy, PTG showed greater improvement with treatment. Patients 1, 2, 3 and 4, who had very high pre-treatment resilience levels, showed more significant PTG improvements after treatment than did the patients with lower pre-treatment resilience scores.

\section{DISCUSSION}

After EMDR therapy, seven subjects showed significant increases in PTGI and SRGS scores, which veri- fied that EMDR therapy can facilitate PTG. Not all post-traumatic individuals experience PTG. ${ }^{3)}$ Approximately $15 \%$ of subjects with traumatic experiences suffer from PTSD, and the remaining $85 \%$ show resilient recovery to pre-trauma levels. ${ }^{19)}$ Some show a higher PTG level than before the trauma. Therefore, it was encouraging that seven subjects showed better PTG-related scale scores after EMDR therapy. EMDR therapy helped to facilitate PTG, despite the use of a regular EMDR protocol that was not specifically modified to improve PTG.

During the 8 sessions of EMDR, the reduction in CAPS score was inconsistent across subjects. However, PTGI and SRGS scores increased continuously with repeated sessions. This result is consistent with those of a previous study that confirmed that PTG was gradually facilitated and accumulated regardless of the presence of short-term stress or environmental factors. ${ }^{2)}$

The ESs of PTGI and SRGS were more significant than that of CAPS. The therapeutic effects of EMDR were more specific to PTG facilitation than to PTSD relief. Reasons why the mean decrease in CAPS score was not significant may be that: 1) subjects without PTSD had low CAPS baseline scores at pre-treatment; consequently, they had low decrease rates; 2) the CAPS score of one subject (patient 8) who suffered severe PTSD actually increased, which attenuated the mean decrease in CAPS score for all subjects.

Therapeutic response to EMDR is defined as a CAPS score decrease of $30 \%$ or more ${ }^{15)}$ Four subjects (patients $1,2,3$, and 4) were not diagnosed with PTSD, but showed a $30 \%$ or greater reduction in CAPS score. However, two subjects (patients 6 and 8) who were diagnosed with PTSD at pre-treatment showed a smaller reduction or no reduction in CAPS score. The reason for the poorer PTSD relief in these two subjects may have been an insufficient number of EMDR sessions to address these subjects' severe and consistent PTSD symptoms. EMDR is typically administered for six to 12 sessions, but can be administered for more than 12 sessions when improvements in symptoms are non-significant or when symptoms are severe. ${ }^{20)}$ When symptoms are severe, EMDR treatments are often accompanied by medication. ${ }^{20)}$ In this study, only EMDR therapy was applied, which might have been why therapeutic responses were delayed.

CD-RISC scores reflect individual resilience. ${ }^{17)}$ Resilience is defined as the power or ability of individuals to overcome adversity and to recover their adaptation to levels similar to those pre-stress. ${ }^{21)}$ The opposite concept of resilience is vulnerability. Resilience should be measured 
and evaluated to anticipate the possibility of individual positive post-traumatic changes and their degree. Individuals with higher resilience levels are expected to experience better PTG facilitation, and vice versa. In this study, subjects with higher pre-treatment resilience levels (patients 1, 2, 3, and 4) showed more significant PTG improvement after treatment than did those with lower pre-treatment resilience levels.

All subjects except patient 8 showed improvement in resilience level after EMDR therapy. Resilience is simple functional recovery to pre-traumatic levels, ${ }^{21)}$ whereas PTG is change and growth that exceeds resilience. ${ }^{3)}$ In other words, PTG is an inclusive concept that encompasses resilience. PTG improvement in the seven subjects observed in this study also indicates improvement in resilience as a matter of course.

Three subjects (patients 5, 6, and 7) showed meaningful changes. Although the reductions in CAPS score were not significant, the increases in PTGI and SRGS scores were significant, which indicates minor PTSD improvement, but major PTG facilitation. No consistent association between PTG and PTSD has been observed in previous cross-sectional studies, and the results tend to be contradictory. ${ }^{22)}$ Although PTG and PTSD are opposite concepts, PTG does not always indicate relief of PTSD. If individuals develop philosophical or further changes in emotional, psychological, and physical health, the case is one of PTG, regardless of adaptation level and post-traumatic distress. ${ }^{3)}$ This means that PTSD can remain even if post-traumatic PTG is achieved. The patterns of change observed in patients 5,6 , and 7 were consistent with this relationship between PTG and PTSD and imply that EMDR can be separately effective for improving PTG, regardless of PTSD.

In cross-sectional studies, the relationship between PTG and PTSD has been shown to be complex, whereas longitudinal studies have shown consistent correlations between the two. In most longitudinal studies, a strong correlation between PTG and PTSD was observed approximately two years after trauma. ${ }^{23,24)}$ PTG did not appear to be associated with PTSD relief over the short term, but subjects with high PTG level showed high levels of adaptation two years after trauma. PTG facilitation appears to contribute to PTSD relief over the long term. Therefore, therapeutic approaches to treat patients after trauma should focus on PTG. In this study, PTG improved after treatment, but PTSD was not significantly relieved in patients 5,6, and 7. Therefore, re-evaluation and monitoring may be needed for a long time after treatment in such patients. In patients 1, 2, 3, and 4, PTG was strongly facilitated from the start, and PTSD was abruptly relieved. These patients showed ideal results of PTSD relief and PTG facilitation through EMDR therapy.

Patient 8 who had comorbid MDD did not show a reduction in CAPS score after EMDR but did not show increases in PTGI, SRGS, and CD-RISC scores. Patient 8 felt guilt over the deaths of her colleagues and abnormal bereavement, which led to EMDR having almost no therapeutic effect. This patient's unstable familial support system and low socioeconomic status might also have contributed to this outcome. The resilience level of this patient was especially low before EMDR treatment, and her vulnerability to trauma was significant. Time is the most important intervention factor in PTG, but comorbid psychiatric disorder, psychological resources, severity of the traumatic events, and social/familial support are also important. ${ }^{25,26)}$ When a patient have a comorbid depression, and shows vulnerability and lack of psychological resources, treatment interventions to facilitate PTG may be ineffective. Patient 8 may therefore require longer-term treatment or combined treatment.

CAPS, PTGI, and SRGS scores decreased or increased continuously between time point of EMDR beginning and end session. These results provide an indication of 'treatment outcome'. It means that EMDR produces significant treatment gains (reduction of PTSD level and improvement of PTG) with only 8-EMDR sessions. On the other hand, their scores reached a plateau from time point of session 8 to three months follow-up. These results provide an indication of 'maintenance effect'. Overall it appears that end-point treatment gains appear to have been relatively well maintained at follow-up. However, presentation of only three-months follow-up results may provide an over-optimistic picture of long-term treatment gains.

The limitations of this study are as follows. 1) The relatively small sample size limits the generalizability of our results. Group therapies were not possible due to the characteristics of EMDR, and clinician resources and time were limited. 2) There was no control group that was not treated by EMDR. 3) Five of eight subjects were adolescents, and the three adult subjects were of different ages and had different backgrounds and occupations. 4) PTG results should be monitored on a long-term basis; 10- or 11-month follow-up observations are not necessarily sufficient to evaluate PTG improvements. Nevertheless, this study is meaningful because a single clinician consistently applied EMDR, and the subjects experienced the same trauma type, period, and severity. In addition, PTG has pre- 
viously been studied mostly in the form of group psychotherapy; in this study, EMDR was applied individually.

Clinical application of PTG does not require rejection of PTSD and does not mean that treatment of post-traumatic psychological distress is unnecessary. Beyond the negative perspectives of PTSD, the concept of PTG must always be reflected in post-traumatic treatment. It is important to integrate PTG into typical treatments for trauma rather than suggesting new types of treatment. Most previous studies were not designed to facilitate PTG as primary goal, but to confirm PTG improvement as a byproduct of PTSD treatment (not primary outcome but secondary outcome). EMDR is a reliable method for treating traumatic memories. EMDR can be effective at treating negative aspects (relief of PTSD) and inducing positive changes (facilitation of PTG).

Studies of conditions that facilitate PTG are not sufficient to provide patients with customized treatments. With a better understanding of PTG, the conditions of growth may be clarified, and the best way to facilitate growth may be identified.

\section{Acknowledgments}

This research was supported by a grant of the Korea Health Technology R\&D Project through the Korea Health Industry Development Institute (KHIDI), funded by the Ministry of Health \& Welfare, Republic of Korea (HC15C1405).

\section{REFERENCES}

1. Brewin CR, Holmes EA. Psychological theories of posttraumatic stress disorder. Clin Psychol Rev 2003;23:339-376.

2. Tedeschi RG, Calhoun LG. The Posttraumatic Growth Inventory: measuring the positive legacy of trauma. $J$ Trauma Stress 1996;9:455-471.

3. Tedeschi RG, Calhoun LG. Beyond the concept of recovery: growth and the experience of loss. Death Stud 2008;32: 27-39.

4. Bonanno GA. Loss, trauma, and human resilience: have we underestimated the human capacity to thrive after extremely aversive events? Am Psychol 2004;59:20-28.

5. Ramos C, Leal I, Tedeschi RG. Protocol for the psychotherapeutic group intervention for facilitating posttraumatic growth in nonmetastatic breast cancer patients. BMC Womens Health 2016;16:22.

6. Wagner AC, Torbit L, Jenzer T, Landy MS, Pukay-Martin ND, Macdonald A, et al. The role of posttraumatic growth in a randomized controlled trial of cognitive-behavioral conjoint therapy for PTSD. J Trauma Stress 2016;29: 379-383.

7. Shapiro F, Maxfield L. Eye movement desensitization and reprocessing (EMDR): information processing in the treatment of trauma. J Clin Psychol 2002;58:933-946.

8. Davidson PR, Parker KC. Eye movement desensitization and reprocessing (EMDR): a meta-analysis. J Consult Clin Psychol 2001;69:305-316.

9. Tesarz J, Leisner S, Gerhardt A, Janke S, Seidler GH, Eich $\mathrm{W}$, et al. Effects of eye movement desensitization and reprocessing (EMDR) treatment in chronic pain patients: a systematic review. Pain Med 2014;15:247-263.

10. Gielkens EM, Sobczak S, Van Alphen SP. Eye movement desensitization and reprocessing therapy for personality disorders in older adults? Int Psychogeriatr 2016;28:17511752.

11. Shapiro S. Enhancing self-belief with EMDR: developing a sense of mastery in the early phase of treatment. Am J Psychother 2001;55:531-542.

12. Lytle RA, Hazlett-Stevens H, Borkovec TD. Efficacy of eye movement desensitization in the treatment of cognitive intrusions related to a past stressful event. J Anxiety Disord 2002;16:273-288.

13. Shapiro F. Eye movement desensitization and reprocessing (EMDR) and the anxiety disorders: clinical and research implications of an integrated psychotherapy treatment. J Anxiety Disord 1999;13:35-67.

14. Park CL, Cohen LH, Murch RL. Assessment and prediction of stress-related growth. J Pers 1996;64:71-105.

15. Blake DD, Weathers FW, Nagy LM, Kaloupek DG, Gusman FD, Charney DS, et al. The development of a ClinicianAdministered PTSD Scale. J Trauma Stress 1995;8:75-90.

16. Lee BY, Kim Y, Yi SM, Eun HJ, Kim DI, Kim JY. A reliability and validity study of a clinician-administered PTSD scale. J Korean Neuropsychiatr Assoc 1999;38:514-522.

17. Connor KM, Davidson JR. Development of a new resilience scale: the Connor-Davidson Resilience Scale (CD-RISC). Depress Anxiety 2003;18:76-82.

18. Baek HS, Lee KU, Joo EJ, Lee MY, Choi KS. Reliability and validity of the Korean version of the connor-davidson resilience scale. Psychiatry Investig 2010;7:109-115.

19. Breslau N. The epidemiology of posttraumatic stress disorder: what is the extent of the problem? J Clin Psychiatry 2001;62 Suppl 17:16-22.

20. National Collaborating Centre for Mental Health (UK). Post-traumatic stress disorder: The management of PTSD in adults and children in primary and secondary care. Leicester (UK):Gaskell;2005.

21. Southwick SM, Bonanno GA, Masten AS, Panter-Brick C, Yehuda R. Resilience definitions, theory, and challenges: interdisciplinary perspectives. Eur J Psychotraumatol 2014; 5:10.3402/ejpt.v5.25338.

22. Frazier $\mathrm{P}$, Conlon A, Glaser T. Positive and negative life changes following sexual assault. J Consult Clin Psychol 2001;69:1048-1055.

23. Davis CG, Nolen-Hoeksema S, Larson J. Making sense of loss and benefiting from the experience: two construals of meaning. J Pers Soc Psychol 1998;75:561-574.

24. Ickovics JR, Meade CS, Kershaw TS, Milan S, Lewis JB, Ethier KA. Urban teens: Trauma, posttraumatic growth, and emotional distress among female adolescents. J Consult Clin Psychol 2006;74:841-850.

25. Danhauer SC, Case LD, Tedeschi R, Russell G, Vishnevsky $\mathrm{T}$, Triplett $\mathrm{K}$, et al. Predictors of posttraumatic growth in women with breast cancer. Psychooncology 2013;22: 2676-2683.

26. Kwon A, Song J, Yook KH, Jon DI, Jung MH, Hong N, et al. Predictors of suicide attempts in clinically depressed Korean adolescents. Clin Psychopharmacol Neurosci 2016; 14:383-387. 